\title{
Análise da contribuição das FinTechs para o crescimento econômico na América Latina no período de 2008 a 2018 à luz do modelo schumpeteriano
}

Fábio Cossenzo ${ }^{1}$

\begin{abstract}
RESUMO
Uma das maneiras mais importantes de que os diversos países do mundo dispõem para evitar a pobreza e melhorar a qualidade de vida da população é promovendo o seu crescimento econômico. Na América Latina, os países ainda não alcançaram o nível de países desenvolvidos, de modo que esta temática é de maior relevância. Desde 2008, com o surgimento das FinTechs, a indústria financeira sofreu uma disrupção tecnológica, que remonta ao conceito de "destruição criativa" de Joseph Schumpeter, tornando interessante a modelagem do crescimento econômico e o impacto trazido pelas inovações das FinTechs por meio de um modelo endógeno schumpeteriano de crescimento econômico. O objetivo geral do trabalho é analisar como o PIB per capita, PPC (dólar), dos países selecionados da América Latina (Colômbia, Peru, México, Argentina, Chile e Brasil) foi influenciado pelos fatores relacionados ao surgimento de FinTechs nesses países após a crise financeira global de 2008. Os resultados apontam para a utilidade do modelo econométrico proposto, pois o modelo foi considerado apropriado pelos testes de significância para os seis países da América Latina observados. Dentre os fatores relacionados ao surgimento de FinTechs, os mais relevantes para o crescimento econômico dos países foram o inverso do tempo médio de existência das FinTechs sediadas no país, a quantidade de FinTechs sediadas no país e a quantidade de FinTechs voltadas para a tecnologia de inteligência artificial.
\end{abstract}

Palavras-chave: crescimento econômico; modelo schumpeteriano; fintech; inovação.

\section{Analysis of FinTechs' contribution to economic growth in Latin America from 2008 to 2018 based on Schumpeterian model}

\begin{abstract}
One of the most important ways in which countries have at their disposal to avoid poverty and improve population welfare is by promoting their economic growth. In Latin America, countries have not yet achieved the level of developed countries, so this issue is of greater relevance. Since 2008, with the emergence of FinTechs, the financial industry has experienced a technological disruption, which recalls the "creative destruction" concept by Joseph Schumpeter, so that it becomes interesting to model the economic growth and the related FinTechs' impacts through a Schumpeterian endogenous economic growth model. The main objective of this work is to analyze how GDP per capita PPP (USD) of select countries from Latin America (Colombia, Peru, Mexico, Argentina, Chile and Brazil) was influenced by factors related to the emergence of FinTechs in these countries after the global financial crisis of 2008. The results point to the usefulness of the proposed econometric model, as the model was considered appropriate by the significance tests for the six Latin American countries observed. Among the factors related do the emergence of FinTechs, the most relevant factors to the countries' economic growth were the inverse of the average time of existence of FinTechs headquartered in the country, the number of FinTechs headquartered in the country and the number of FinTechs focused on artificial intelligence technology.
\end{abstract}

Keywords: economic growth; schumpeterian model; fintech; innovation.

\section{INTRODUÇÃO}

Uma das maneiras mais importantes de que os diversos países do mundo dispõem para evitar a pobreza e melhorar a qualidade de vida da população é promovendo o seu crescimento econômico. Na América Latina, os países ainda não alcançaram o nível de países desenvolvidos, de modo que esta temática é de maior relevância.

\footnotetext{
${ }^{1}$ Bacharel em Ciência da Computação pela Universidade Federal de Minas Gerais e Bacharel em Ciências Econômicas pela Universidade Paulista. Pós-graduando em Inteligência Artificial e Aprendizado de Máquina pela Pontifícia Universidade Católica de Minas Gerais. Possui experiência em Supervisão Prudencial e de Conduta de Instituições Financeiras, Gestão de Equipes, Tecnologia da Informação, FinTech, Gestão de Projetos, Gestão de Informações e Gestão de Processos de Negócio.
} 
Na moderna teoria do crescimento, o modelo de Robert Solow possui papel central, todavia é um modelo que não responde plenamente algumas questões fundamentais, tal como o motivo para a renda per capita ser tão maior nos países membros da Organização para a Cooperação e Desenvolvimento Econômico (OCDE) do que nos países menos desenvolvidos do mundo, como os da América Latina.

Desde 2008, com o surgimento das FinTechs - que representam a junção de "finance" e "information technology" -, a indústria financeira sofreu uma disrupção tecnológica, um significativo salto em inovação, onde há oportunidades para a criação de novos serviços e modelos de negócios e desafios para os tradicionais provedores de serviços financeiros.

Neste contexto, torna-se importante investigar a contribuição das FinTechs para o desenvolvimento do sistema financeiro e, por conseguinte, para o crescimento econômico dos países onde estas instituições estão sediadas.

As FinTechs podem representar uma disrupção do mercado financeiro capaz de reposicionar os países no ranking do desenvolvimento econômico. Tendo em vista que essa disrupção remonta ao conceito de "destruição criativa" de Schumpeter, torna-se interessante a modelagem do crescimento econômico e o impacto trazido pelas inovações das FinTechs por meio de um modelo endógeno schumpeteriano de crescimento econômico.

Neste trabalho de pesquisa pretende-se analisar a contribuição das FinTechs para o crescimento econômico de países selecionados da América Latina no período de 2008 a 2018 sob o prisma do modelo de crescimento econômico endógeno schumpeteriano de um setor descrito por Aghion e Howitt (2009, p. 95).

O enfoque desta pesquisa é a região da América Latina e, deste conjunto de países, foram selecionados aqueles que tinham maior relevância no uso de serviços oferecidos por FinTechs. Desse modo, adotou-se como critério de seleção o ranking de países da América Latina com maior uso de serviços de FinTechs pelos consumidores segundo o Índice Global de Adoção de FinTechs de 2019, elaborado pela Ernst \& Young (2019).

Portanto, nos experimentos deste trabalho, para fins de análise e comparação, foram considerados na amostra de países Colômbia, Peru, México, Argentina, Chile e Brasil, os quais representavam cerca de 91\% do mercado de FinTechs da América Latina em 2018.

\section{FUNDAMENTAÇÃO TEÓRICA}

\subsection{Conceito de Crescimento Econômico}

A pobreza é o problema básico que os países em desenvolvimento enfrentam (KRUGMAN, OBSTFELD e MELITZ, 2015, p. 523, tradução nossa), como é o caso da América Latina. Segundo Jones e Vollrath (2015), uma das maneiras de tornar esses países ricos é promovendo o seu crescimento econômico, cujas taxas, de modo geral, não permanecem constantes ao longo do tempo e podem variar substancialmente de um país para o outro, até mesmo alterando a posição relativa de um país na distribuição mundial da renda per capita. Ou seja, por meio do crescimento econômico é possível que os países em desenvolvimento alterem, de modo significativo, seu posicionamento no ranking de PIB per capita, o que poderia levar a melhores condições socioeconômicas da população.

Cabe aqui trazer uma definição de crescimento econômico que se pretende adotar neste trabalho, bem como diferenciá-la do conceito de desenvolvimento econômico, para fins de melhor compreensão dos objetivos e dos resultados desta pesquisa.

Antes de iniciar, vale fazer um breve esclarecimento sobre a dicotomia "crescimento" versus "desenvolvimento" econômico, que tem gerado polêmica recente dentro e fora da academia. Antes de tudo, no mundo acadêmico, a distinção entre "teorias de crescimento" e "teorias de desenvolvimento" é relacionada ao objeto de estudo em questão. Segundo a definição mais usual, as primeiras teriam como alvo o entendimento do fenômeno do crescimento de modo geral, enquanto os estudiosos do "desenvolvimento" estariam mais centrados em entender diferenças entre os países. (...)

Porém a conotação mais comum dada à dicotomia é outra. Críticos das medidas tradicionais de crescimento (e nível) do PIB por habitante enfatizam que essa medida desconsidera outras variáveis também relevantes para o bem-estar econômico-social de

Revista Desenvolvimento Socioeconômico em debate v.7 n.2 (2021) 
uma nação. A crítica é válida, e os principais manuais de teoria econômica de fato enfatizam a diferença entre nível de renda e bem-estar social (...). Porém, em média, e considerando períodos mais longos, a variável PIB por habitante não é má aproximação para um índice composto de desenvolvimento social que incorpore essas outras dimensões. (FERREIRA, P. C. et al., 2013, p. 39).

De modo mais específico, Brue e Grant (2016, p. 505) conceituam o crescimento econômico como "o aumento da produção real de um país (PIB)" ou "PIB real per capita", que resulta, dentre outros fatores, de "avanços tecnológicos que impulsionam a produtividade". Esse é o conceito utilizado neste trabalho.

\subsection{Desenvolvimento Financeiro e Crescimento Econômico}

Em 1911, Joseph A. Schumpeter argumentou que os serviços providos por intermediários financeiros são essenciais para a inovação tecnológica e o desenvolvimento econômico (KING e LEVINE, 1993). Há inúmeros estudos que afirmam e evidenciam que há uma robusta correlação entre o desenvolvimento financeiro e o crescimento econômico (HAMDI, HAKIMI e SBIA, 2017; PHILIPPON, 2016; KRUGMAN, OBSTFELD e MELITZ, 2015, p. 523-527; AGHION e DURLAUF, 2005; KING e LEVINE, 1993).

Hamdi, Hakimi e Sbia (2017) realizaram uma pesquisa com o objetivo de investigar a relação entre o desenvolvimento do setor financeiro e o crescimento econômico, levando em consideração o papel da qualidade das instituições. Utilizaram uma amostra de 143 países observados durante o período de 2006 a 2013. A amostra foi dividida em 100 países em desenvolvimento e 43 países desenvolvidos. $\mathrm{O}$ artigo mostrou que o setor financeiro executa um papel crucial no desenvolvimento e crescimento econômico para a amostra completa, assim como para os grupos de países desenvolvidos e em desenvolvimento.

Entretanto, os resultados obtidos por Hamdi, Hakimi e Sbia (2017) indicaram que os países desenvolvidos se beneficiam mais da presença de instituições adequadas em seus países, as quais, portanto, tiveram maior contribuição para o crescimento econômico daqueles países.

Essa constatação reforça a importância de se apoiar novas formas de promover o crescimento econômico em países em desenvolvimento, como é o caso da América Latina, pois o fortalecimento e aprimoramento de instituições já consolidadas têm menor contribuição para o aumento do PIB per capita nesses países.

\subsection{Crescimento Econômico na América Latina}

O crescimento econômico tem sido frustrante na maioria dos países latino-americanos, havendo uma necessidade de políticas produtivas ativas, e uma necessidade ainda maior de políticas tecnológicas ativas, as quais foram negligenciadas durante a fase de industrialização dirigida pelo Estado (OCAMPO e FLORES, 2015, p. 328).

Segundo um relatório da OCDE (OECD et al., 2019), no período de 2008 a 2018, o PIB per capita médio dos países do G7 permaneceu cerca de três vezes maior do que o dos países da América Latina e Caribe. Além disso, o contexto internacional apresenta vários riscos à região.

A redução do ritmo de crescimento do PIB mundial, guerras comerciais e alterações da política de juros dos Estados Unidos são elementos que podem levar a dificuldades para o crescimento do PIB da América Latina.

Adicionalmente, o referido relatório da OCDE ainda avalia que é limitado o espaço para políticas de aumento da demanda que possam impulsionar o crescimento. O espaço fiscal é restrito haja vista que os superávits primários estão aquém dos níveis necessários para estabilização das dívidas. E, embora a inflação esteja sob controle na maioria dos países da América Latina e do Caribe, há reduzido espaço para adoção de política monetária expansionista.

A conclusão do relatório é que o desempenho econômico da América Latina e Caribe é limitado por fatores estruturais.

Nesse sentido, um caminho possível para lidar com as dificuldades enfrentadas seria o investimento em soluções inovadoras para reduzir desigualdades, melhorar o bem-estar da população e reconstruir a confiança nas instituições, tanto domesticamente como também em nível multilateral. Essa proposta leva 
em consideração que atualmente (2019) se percebe um rápido progresso tecnológico e um processo de acelerada digitalização de serviços.

Portanto, tendo em vista os desafios a serem enfrentados pelos países da América Latina, torna-se importante avaliar outros modelos de crescimento econômico para essa região.

\subsection{Modelo Schumpeteriano de Crescimento Econômico}

Na moderna teoria do crescimento, o modelo de Robert Solow possui papel central, todavia é um modelo que não responde plenamente algumas questões fundamentais, tais como os motivos para: o crescimento médio na renda per capita ser tão maior agora do que era 200 anos atrás, e a renda per capita ser tão maior nos países membros da OCDE do que nos países menos desenvolvidos do mundo. Esta fragilidade no modelo de Solow - e os modelos dele derivados - foi a principal razão para o desenvolvimento da classe de modelos de crescimento endógeno (AGHION e DURLAUF, 2005, p. 15).

Dentre a classe de modelos de crescimento endógeno, encontra-se o modelo schumpeteriano. De acordo com Resende e Gonçalves (2006), o modelo schumpeteriano de crescimento endógeno considera o progresso técnico como fundamental determinante do crescimento econômico.

Segundo Shikida e Bacha (1998), as ideias schumpeterianas e neo-schumpeterianas mostraram-se adequadas para avançar, teórica e empiricamente, na discussão sobre os determinantes do processo dinâmico de desenvolvimento, da mudança tecnológica e da inovação.

Em seu trabalho sobre a "A natureza da inovação financeira: uma análise pós-schumpeteriana", Akdere e Benli (2018) se perguntam se é possível aplicar uma noção de inovação empresarial schumpeteriana para a esfera financeira. Nessa pesquisa, relatam que Schumpeter teria restringido essa análise ao setor real da economia. No entanto, no referido trabalho, Akdere e Benli demonstram que a análise de inovação empresarial de Schumpeter também fornece um arcabouço teórico para o estudo entre inovações empresariais e inovações financeiras.

Aghion (2004) argumenta que a abordagem schumpeteriana para o crescimento econômico é microfundamentada e fornece as ferramentas analíticas para projetar com sucesso estratégias e instituições apropriadas para alcançar rápida convergência e crescimento sustentável em países em diferentes níveis iniciais de desenvolvimento tecnológico. Três ideias básicas sustentariam essa abordagem schumpeteriana: (i) a principal fonte de progresso tecnológico é a inovação; (ii) inovações são criadas por empresas que visam lucro no caso de sucesso de sua implementação; (iii) a noção de "destruição criativa", ou seja, novos processos ou produtos introduzidos pelos inovadores atuais se tornam obsoletos quando outras inovações competem com as tecnologias atuais, retirando-as do mercado. No mesmo artigo, Aghion desenvolve o modelo básico de crescimento schumpeteriano e o utiliza para analisar os determinantes institucionais de crescimento no longo prazo.

Posteriormente, Aghion e Howitt (2009) desenvolvem um modelo de crescimento endógeno, no qual o crescimento é gerado por uma sequência aleatória de inovações de melhoria da qualidade (ou "verticais"), com base na moderna teoria da organização industrial, que retrata a inovação como uma importante dimensão da competição industrial. O modelo é chamado schumpeteriano porque incorpora a força que Schumpeter (1943) chamou de "destruição criativa".

\section{PROCEDIMENTOS METODOLÓGICOS}

Esta é uma pesquisa explicativa sobre o crescimento econômico à luz do modelo schumpeteriano, com análise de dados sobre PIB per capita e fatores relacionados ao surgimento de FinTechs em países da América Latina, observando-se o período de 2008 a 2018.

\subsection{Universo e Amostra}

O universo é composto pelos dados sobre o crescimento econômico (PIB per capita) e os fatores relacionados ao surgimento de FinTechs em países da América Latina, observando o período de 2008 a 2018, ou seja, desde o surgimento das FinTechs (2008) até a data mais recente em que há dados disponíveis sobre o PIB per capita (2018). 
O enfoque desta pesquisa é a região da América Latina e, deste conjunto de países, foram selecionados aqueles que tinham maior relevância no uso de serviços oferecidos por FinTechs. Desse modo, adotou-se como critério de seleção o ranking de países da América Latina com maior uso de serviços de FinTechs pelos consumidores segundo o Índice Global de Adoção de FinTechs de 2019, elaborado pela Ernst \& Young (2019).

Portanto, nos experimentos deste trabalho, para fins de análise e comparação, foram considerados na amostra de países da América Latina: Colômbia, Peru, México, Argentina, Chile e Brasil.

\subsection{Coleta de Dados}

Representando os dados sobre o crescimento econômico foi utilizada a base de dados do Banco Mundial (2019) denominada "GDP per capita, PPP (current international \$)", identificada por "NY.GDP.PCAP.PP.CD". Essa base de dados se refere ao PIB per capita baseado na paridade do poder de compra (PPC). O PIB per capita é convertido para dólares internacionais, os quais têm o mesmo poder de compra que o dólar americano (USD) nos Estados Unidos.

Os fatores relacionados ao surgimento de FinTechs na América Latina foram obtidos de uma base de dados da Crunchbase Inc. (2019) de 832 empresas da categoria "FinTech" até a data de 22 de outubro de 2019 (data da consulta dos dados) e que são sediadas nos seguintes países: Argentina, Brasil, Colômbia, Chile, México e Peru. Ainda foram definidos, como filtros, as categorias "FinTech", "Blockchain" e "Artificial Intelligence". Desse conjunto, foram deduzidas 109 FinTechs sem data de fundação (informação essencial para o experimento), 7 FinTechs fechadas sem data de encerramento das atividades (informação também essencial para o experimento) e 22 FinTechs fundadas em 2019 (fora do intervalo de estudo, que se restringe ao período de 2008 a 2018). Desse modo, ao final foram selecionadas 694 FinTechs na amostra.

\subsection{Descrição do Modelo Teórico}

Nesta pesquisa será utilizado como base um modelo endógeno de crescimento econômico schumpeteriano de um setor descrito por Aghion e Howitt (2009, p. 95), no qual o PIB per capita é proporcional a um parâmetro de produtividade $A_{t}$. Esse modelo é adequado para o presente estudo, pois este visa estudar o aumento de produtividade atrelado ao surgimento das FinTechs em determinado país que possa contribuir para o seu crescimento econômico. Foster e Rosenweig (2010) já afirmavam que diferenças nos níveis tecnológicos entre países representam um grande componente nas diferenças de PIB per capita.

No caso deste trabalho, o aumento de produtividade se daria por meio dos fatores relacionados ao surgimento de FinTechs no país, ou seja, "o PIB per capita é proporcional aos fatores relacionados ao surgimento de FinTechs no país, os quais estariam atrelados ao aumento da produtividade".

\subsection{Especificação do Modelo Econométrico}

Neste trabalho será realizada uma análise econométrica, baseada na teoria econômica de crescimento endógeno schumpeteriano e no modelo matemático endógeno schumpeteriano de crescimento econômico de um setor descrito por Aghion e Howitt (2009, p. 95).

O modelo econométrico é descrito da seguinte forma:

$$
\text { Crescimento }_{i, t}=\beta_{0}+\beta_{1} \text { FinTechs }_{i, t}+\beta_{2} \text { Blockchain }_{i, t}+\beta_{3} I A_{i, t}+\beta_{4} T M E_{i, t}+\varepsilon_{i, t}
$$

O PIB per capita é denotado por Crescimento $_{i_{i},}$, que é a variável dependente. As variáveis explicativas são os fatores relacionados ao surgimento de FinTechs em cada um dos países que se desejam estudar. 
A quantidade de FinTechs sediadas no país é representada por FinTechs $s_{i, t}$, a quantidade de FinTechs da categoria "Blockchain" é representada por Blockchain $n_{i, t}$ e, finalmente, a quantidade de FinTechs da categoria "Inteligência Artificial" é representada por $I A_{i, t}$.

A variável $T M E_{i, t}$ representa a razão (1/tempo médio de existência das FinTechs sediadas no país), considerando que um menor tempo médio de existência das FinTechs indicaria maior fluxo de entrada de novos players no mercado, aumentando a competição e a oferta de produtos e serviços financeiros por meio de tecnologias inovadoras.

\section{O ADVENTO DAS FINTECHS}

\subsection{Evolução da tecnologia financeira}

Desde 2008, a indústria financeira sofreu uma evolução tecnológica marcada pelo surgimento das "FinTechs" (Quadro 1), que representam a união de "finance" e "information technology", gerando oportunidades para a criação de novos serviços e modelos de negócios e impondo desafios para os tradicionais provedores de serviços financeiros (ZAVOLOKINA, DOLATA e SCHWABE, 2016).

Conforme destacam Alt, Beck e Smits (2018), as FinTechs detêm um potencial disruptivo para a indústria financeira e vão além de uma simples continuação linear dos desenvolvimentos existentes, o que remonta ao conceito de "destruição criativa" de Schumpeter (AGHION, 2004, p. 8).

Quadro 1 - Evolução da tecnologia financeira.

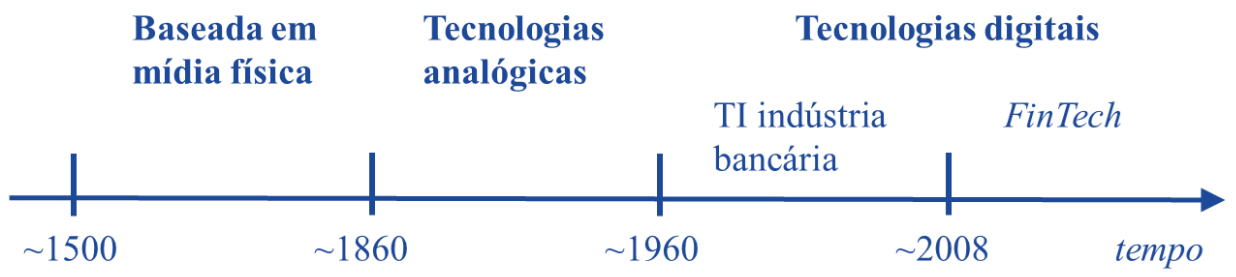

Fonte: Alt, Beck e Smits (2018, p. 236).

Na Tabela 1 é apresentada uma comparação entre a Tecnologia da Informação (TI) da indústria bancária tradicional (até por volta de 2008) e a FinTech (a partir de 2008) segundo três níveis de transformação: (i) ambiente externo, (ii) rede de instituições e (iii) organização interna das instituições. Observa-se que as instituições, para lograrem êxito neste novo cenário, precisam alterar de forma substancial seus processos internos e a forma como interagem com clientes e com o mercado.

Tabela 1 - Comparação entre a TI bancária tradicional e a FinTech.

\begin{tabular}{|l|l|l|l|}
\hline $\begin{array}{l}\text { Nível de } \\
\text { transformação }\end{array}$ & Aspecto observado & TI indústria bancária & FinTech \\
\hline Ambiente externo & Regulação & $\begin{array}{l}\text { Baixa exigência de capital; } \\
\text { pouca supervisão }\end{array}$ & $\begin{array}{l}\text { Regras mais estritas; menos } \\
\text { proteção }\end{array}$ \\
\cline { 2 - 4 } & $\begin{array}{l}\text { Inovação do } \\
\text { modelo de negócios }\end{array}$ & $\begin{array}{l}\text { Ramo específico do negócio; } \\
\text { serviços off-line }\end{array}$ & Serviços on-line e móveis \\
\cline { 2 - 4 } & $\begin{array}{l}\text { Governança das } \\
\text { infraestruturas }\end{array}$ & $\begin{array}{l}\text { Instituição centralizada como } \\
\text { uma firma local }\end{array}$ & Distribuição de tarefas \\
\cline { 2 - 4 } & $\begin{array}{l}\text { Estilo de } \\
\text { pagamento }\end{array}$ & $\begin{array}{l}\text { Maioria dos clientes utilizando } \\
\text { dinheiro em espécie }\end{array}$ & $\begin{array}{l}\text { Aumento dos pagamentos sem } \\
\text { uso de dinheiro em espécie }\end{array}$ \\
\hline $\begin{array}{l}\text { Redacionamento de } \\
\text { instituições }\end{array}$ & $\begin{array}{l}\text { Pequeno número de parceiros } \\
\text { entre as }\end{array}$ & Muitos parceiros especializados \\
\hline
\end{tabular}




\begin{tabular}{|c|c|c|c|}
\hline & $\begin{array}{l}\text { Margens e } \\
\text { estrutura de custos }\end{array}$ & $\begin{array}{l}\text { Margens elevadas no core } \\
\text { business }\end{array}$ & $\begin{array}{l}\text { Margens reduzidas; maior } \\
\text { competição }\end{array}$ \\
\hline & Competidores & $\begin{array}{l}\text { Outros provedores de serviços } \\
\text { financeiros tradicionais }\end{array}$ & Start-ups; entrantes laterais \\
\hline & Cultura & Hierárquica & Cooperativa; ágil \\
\hline & $\begin{array}{l}\text { Retenção de } \\
\text { clientes }\end{array}$ & $\begin{array}{l}\text { Elevado nível de fidelidade dos } \\
\text { clientes }\end{array}$ & $\begin{array}{l}\text { Custos reduzidos para a } \\
\text { mudança de instituição }\end{array}$ \\
\hline \multirow{7}{*}{$\begin{array}{l}\text { Organização } \\
\text { interna das } \\
\text { instituições }\end{array}$} & Foco de negócio & Orientado a processos & Centrado no cliente \\
\hline & $\begin{array}{l}\text { Interação com o } \\
\text { cliente }\end{array}$ & Primeiro off-line & Primeiro on-line; omnicanal \\
\hline & $\begin{array}{l}\text { Competências } \\
\text { essenciais }\end{array}$ & $\begin{array}{l}\text { Distribuição; produtos; } \\
\text { transações }\end{array}$ & $\begin{array}{l}\text { Distribuição on-line; } \\
\text { plataformas }\end{array}$ \\
\hline & Integração vertical & Elevado nível de integração & Baixo nível de integração \\
\hline & $\begin{array}{l}\text { Portfólio de } \\
\text { serviços }\end{array}$ & $\begin{array}{l}\text { Bancos são os provedores } \\
\text { gerais de serviços }\end{array}$ & $\begin{array}{l}\text { Pequenos e diversos provedores } \\
\text { de serviços }\end{array}$ \\
\hline & Automação & $\begin{array}{l}\text { Processos requerem intervenção } \\
\text { manual }\end{array}$ & $\begin{array}{l}\text { Processos completamente } \\
\text { automatizados }\end{array}$ \\
\hline & Arquitetura de TI & $\begin{array}{l}\text { Sistemas monolíticos; } \\
\text { desenvolvimento inhouse }\end{array}$ & $\begin{array}{l}\text { Sistemas modulares; uso de } \\
\text { Application Programming } \\
\text { Interfaces (APIs) }\end{array}$ \\
\hline
\end{tabular}

Fonte: Alt, Beck e Smits (2018, p. 238).

Tendo em vista a complexidade de toda essa mudança, algumas instituições financeiras tradicionais optam por fundar novos negócios dentro dessa nova perspectiva tecnológica, ou seja, FinTechs próprias para atuarem nesse ecossistema, concorrendo com os novos players do mercado e até mesmo com suas próprias instituições bancárias tradicionais.

Outras iniciativas incluem a criação de incubadoras ou aceleradoras de FinTechs, visando o desenvolvimento de novas soluções para o mercado, com possível incorporação futura dessas soluções e start-ups ao negócio principal do conglomerado financeiro.

Ainda, outras instituições bancárias, notadamente as de pequeno e médio portes, adaptam-se ao mundo digital, oferecendo serviços móveis, on-line e com uma visão de negócios centrada no cliente.

Segundo o relatório "Fintech na América Latina 2018: crescimento e consolidação" (BID, BID INVEST e FINNOVISTA, 2019), observa-se que os empreendedores de toda a América Latina estão reagindo às oportunidades trazidas pela evolução e digitalização do setor financeiro. Esses empreendedores estão oferecendo novos serviços e modelos de negócios inovadores em todos os segmentos, com foco especial em atender adequadamente os diferentes clientes e desenvolver propostas de valor adequadas, abrangendo clientes que as instituições bancárias tradicionais não atendiam, e se adaptando melhor às mudanças demográficas e de comportamento dos usuários de produtos e serviços financeiros.

\subsection{As FinTechs na América Latina}

O enfoque desta pesquisa é a região da América Latina e, deste conjunto de países, foram selecionados aqueles que tinham maior relevância no uso de serviços oferecidos por FinTechs. Desse modo, adotou-se como critério de seleção o ranking de países da América Latina com maior uso de serviços de FinTechs pelos consumidores segundo o Índice Global de Adoção de FinTechs de 2019, elaborado pela Ernst \& Young (2019).

Em relação à metodologia aplicada nesse relatório (ERNST \& YOUNG, 2019), foi realizada uma pesquisa com 27.103 consumidores adultos digitalmente ativos, entrevistados pela Internet no período entre 4 fevereiro e 11 de março de 2019. Foram entrevistados consumidores de 27 mercados, os quais foram ordenados de forma decrescente pelo índice de adoção de serviços de FinTechs, conforme disposto na Tabela 2.

Observe que o Brasil foi posicionado no $16^{\circ}$ lugar do ranking, o que pode denotar importante receptividade dos consumidores aos serviços providos por FinTechs.

O índice médio obtido naquele relatório foi de $64 \%$, portanto o Brasil está situado na média dos países observados. 
Os outros países da América Latina listados foram Colômbia (76\%), Peru (75\%), México (72\%), Argentina (67\%) e Chile (66\%), todos posicionados acima do Brasil.

Portanto, nos experimentos deste trabalho, para fins de análise e comparação, foram considerados na amostra de países da América Latina os seguintes: Colômbia, Peru, México, Argentina, Chile e Brasil.

Tabela 2 - Ranking de países em adoção de serviços de FinTechs pelos consumidores*.

\begin{tabular}{|c|c|c|c|c|c|}
\hline Posição & País & Índice & Posição & País & Índice \\
\hline 1 & China & $87 \%$ & 15 & Chile & $66 \%$ \\
\hline 2 & Índia & $87 \%$ & 16 & Brasil & $64 \%$ \\
\hline 3 & Rússia & $82 \%$ & 17 & Alemanha & $64 \%$ \\
\hline 4 & África do Sul & $82 \%$ & 18 & Suécia & $64 \%$ \\
\hline 5 & Colômbia & $76 \%$ & 19 & Suíça & $64 \%$ \\
\hline 6 & Peru & $75 \%$ & 20 & Austrália & $58 \%$ \\
\hline 7 & Holanda & $73 \%$ & 21 & Espanha & $56 \%$ \\
\hline 8 & México & $72 \%$ & 22 & Itália & $51 \%$ \\
\hline 9 & Irlanda & $71 \%$ & 23 & Canadá & $50 \%$ \\
\hline 10 & Reino Unido & $71 \%$ & 24 & Estados Unidos & $46 \%$ \\
\hline 11 & Argentina & $67 \%$ & 25 & Bélgica e Luxemburgo & $42 \%$ \\
\hline 12 & Hong Kong & $67 \%$ & 26 & França & $35 \%$ \\
\hline 13 & Singapura & $67 \%$ & 27 & Japão & $34 \%$ \\
\hline 14 & Coreia do Sul & $67 \%$ & & & \\
\hline
\end{tabular}

Fonte: ERNST \& YOUNG (2019). * O índice médio foi de $64 \%$.

\subsection{O Mercado das Fintechs}

Segundo Gomber e Kock (2017, p. 538), o cenário atual é propício para o desenvolvimento de FinTechs porque os clientes no setor financeiro demandam serviços financeiros inteligentes e fáceis de usar, sem dependência de local e hora e a custos cada vez mais reduzidos. Essa demanda conduz a novos modelos de negócio e conceitos tecnológicos, baseados em Internet, dispositivos móveis e canais digitais on-line para a busca de informações financeiras e a realização de transações financeiras.

Atualmente o mercado de FinTechs está evoluindo rapidamente, tanto na diversificação de serviços quanto em investimentos recebidos, segundo o IOSCO Research Report on Financial Technologies (Fintech) (2017).

Existem diversos tipos ou categorias de FinTechs no mundo, além de diferentes formas de organizar esses segmentos dentro do ecossistema de FinTechs.

Apresenta-se na Tabela 3 a proposta de taxonomia de FinTechs utilizada no relatório "Fintech na América Latina 2018: crescimento e consolidação" (BID, BID INVEST e FINNOVISTA, 2019).

Tabela 3 - Taxonomia de FinTechs.

\begin{tabular}{ll}
\multicolumn{1}{c}{ Categoria } & \multicolumn{1}{c}{ Subcategoria } \\
\hline Pagamentos e remessas & Canais e agregadores de pagamentos \\
& Pagamentos móveis e carteiras eletrônicas \\
& Soluções de pagamento móvel em pontos de venda \\
& Soluções de criptomoeda \\
& Transferências internacionais e remessas \\
& Outros \\
\hline Empréstimos & Empréstimos em balanço a consumidores \\
& Empréstimos em balanço a empresas \\
& Empréstimo P2P a consumidores \\
& Empréstimo P2P a empresas \\
\hline Gestão de finanças empresariais & Soluções de gestão financeira e inteligência de negócios \\
& Soluções de contabilidade digital \\
& Faturamento eletrônico \\
& Soluções de cobrança \\
\hline Gestão de finanças pessoais & Outros \\
\hline
\end{tabular}




\begin{tabular}{ll}
\hline & $\begin{array}{l}\text { Plataformas de comparação } \\
\text { Gestão de dívidas } \\
\text { Outros }\end{array}$ \\
\hline Crowdfunding & $\begin{array}{l}\text { Doações } \\
\text { Recompensa } \\
\text { Imobiliário } \\
\text { Capital }\end{array}$ \\
\hline $\begin{array}{ll}\text { Tecnologias empresariais para instituições } \\
\text { financeiras }\end{array}$ & \\
\hline $\begin{array}{l}\text { Negociação de ativos financeiros e } \\
\text { mercados de capitais }\end{array}$ & \\
\hline Gestão patrimonial & \\
\hline Seguros & $\begin{array}{l}\text { Segurança e identidade digital } \\
\text { Pontuação alternativa, identidade e fraude }\end{array}$ \\
& $\begin{array}{l}\text { Prevenção de fraude e gestão de risco } \\
\text { Pontuação alternativa }\end{array}$ \\
\hline Banco digital & Biometria \\
\hline
\end{tabular}

Fonte: BID, BID INVEST e FINNOVISTA (2019).

Segundo o mesmo relatório, na América Latina houve um aumento de 65,9\% na quantidade de FinTechs de 2017 para 2018. Considerando apenas os países selecionados para o presente estudo, observouse o cenário retratado na Tabela 4.

Tabela 4 - FinTechs na América Latina.

\begin{tabular}{|c|c|c|c|}
\hline País & $\begin{array}{c}\text { Quantidade de } \\
\text { FinTechs em 2018 }\end{array}$ & $\begin{array}{c}\text { Representatividade no mercado } \\
\text { da América Latina }\end{array}$ & $\begin{array}{c}\text { Crescimento da quantidade de } \\
\text { FinTechs em relação a 2017 }\end{array}$ \\
\hline Brasil & 380 & $33 \%$ & $65 \%$ \\
\hline México & 273 & $23 \%$ & $52 \%$ \\
\hline Colômbia & 148 & $13 \%$ & $76 \%$ \\
\hline Argentina & 116 & $10 \%$ & $61 \%$ \\
\hline Chile & 84 & $7 \%$ & $29 \%$ \\
\hline Peru & 57 & $5 \%$ & $256 \%$ \\
\hline Total & 1058 & $91 \%$ & \\
\hline
\end{tabular}

Fonte: BID, BID INVEST e FINNOVISTA (2019, p. 28).

A categoria de FinTechs denominada "Pagamentos e remessas" lidera o mercado na América Latina, seguida das categorias de "Empréstimos" e "Gestão de finanças empresariais".

A forte presença de soluções móveis para pagamentos e remessas se deve, em grande parte, à alta penetração de smartphones na região que, em 2017 , atingiu $67 \%$ da população (GSMA, 2018) e aos altos índices de exclusão, pois estima-se que $45 \%$ dos adultos da região ainda estejam excluídos dos serviços financeiros formais por não terem conta bancária (Banco Mundial, 2017). Por outro lado, a oferta limitada de crédito, que implica em custos de acesso e taxas de juros elevados nos serviços financeiros tradicionais, criou uma oportunidade que levou ao surgimento de inúmeros modelos novos para se obter soluções de financiamento mais eficientes e menos onerosas pelas fintechs. Finalmente, a oferta de soluções de gestão de finanças empresariais reflete uma grande oportunidade na região, devido à importância do setor de pequenas e médias empresas (PMEs), que representa aproximadamente $90 \%$ das empresas da América Latina e do Caribe, cujo desafio é digitalizar-se progressivamente. Diante disso, existe grande necessidade no mercado de ferramentas que ajudem as empresas a melhorar a gestão de seus recursos e a fazer a transição para a digitalização de suas finanças. (BID, BID INVEST e FINNOVISTA, 2019, p. 16)

Portanto, verifica-se que o setor de FinTechs é novo, porém cresce em ritmo acelerado, em particular na América Latina. 


\subsection{Inovações Tecnológicas em Destaque}

Um dos grandes avanços proporcionados pelas FinTechs é a tecnologia Blockchain (AKDERE e BENLI, 2018). A plataforma Blockchain oferece oportunidades de negócios sem precedentes e, devido ao seu protocolo e padrão abertos, os quais possuem recursos para suportar inovações independentes e espontâneas, esta tecnologia pode vir a causar disrupções em uma variedade de indústrias, em especial nos sistemas financeiros (SARKINTUDU, IBRAHIM e ABDWAHAB, 2018).

Nicoletti (2017) explica resumidamente o surgimento e a importância inovadora do Blockchain:

Blockchain nasceu em conexão com o Bitcoin, uma moeda virtual. É essencialmente um banco de dados para gravação de transações de um modo seguro. Blockchain é um banco de dados distribuído, capaz de gerar um livro-razão público de todas as transações, não totalmente armazenado em uma única localização física, mas disperso por uma rede de computadores interconectados.

Blockchain é uma solução descentralizada. Por exemplo, todos os participantes de uma rede P2P possuem uma cópia de todo o conjunto de registros. Consequentemente, não há uma autoridade central. Cada participante da rede pode manipular o livro-razão sem causar incidentes de segurança por intermédio de criptografia e assinaturas digitais. Por meio dessas ferramentas digitais, uma identidade da vida real (não visível) vincula-se a uma identidade criptográfica. Isso é útil para verificar e validar transações. Blockchain e tecnologias disruptivas relacionadas têm chamado muita atenção na indústria financeira. Blockchain é relativamente seguro, transparente e imutável. (NICOLETTI, 2017, p. 126127 , tradução nossa)

Como mencionado por Nicoletti (2017), o berço do Blockchain foi o Bitcoin, uma moeda virtual baseada em uma rede Blockchain, que foi desenhado e proposto pela primeira vez em um artigo de autoria desconhecida, assinado por autor de pseudônimo Satoshi Nakamoto.

Nós propusemos um sistema para transações eletrônicas não baseado em confiança. Nós começamos o arcabouço usual para moedas feito a partir de assinaturas digitais, que provê forte controle de posse, mas é incompleto sem um meio para prevenir o duplo-gasto. Para resolver isso, nós propusemos uma rede peer-to-peer utilizando prova-de-trabalho para gravar um histórico público de transações que rapidamente se tornam computacionalmente impraticável para um hacker alterá-lo, se os nós honestos da rede controlarem a maior parte do poder de processamento. A rede é robusta em sua simplicidade não estruturada. Os nós trabalham todos de uma vez com pouca coordenação. Eles não necessitam ser identificados, haja vista que mensagens não são roteadas para um lugar particular e somente precisam ser entregues em uma base de melhor esforço. Os nós podem deixar e retornar à rede de acordo com sua vontade, aceitando a cadeia prova-de-trabalho como prova do que ocorreu enquanto estiveram fora da rede. Eles votam com seu poder de processamento, expressando sua aceitação de blocos válidos pelo trabalho em sua extensão, ou a rejeição de blocos inválidos pela recusa de trabalhar neles. Quaisquer regras necessárias podem ser exigidas com esse mecanismo de consenso. (NAKAMOTO, 2008, tradução nossa)

Além do Blockchain, outra tecnologia de destaque no contexto das FinTechs é a inteligência artificial, que pode ser definida como "a capacidade de uma máquina imitar o comportamento humano inteligente" ou "a habilidade de um agente em atingir objetivos em uma ampla gama de ambientes", o que imediatamente evoca questões econômicas fundamentais, segundo Aghion, Jones e Jones (2017). No trabalho desses autores são estudadas as implicações da inteligência artificial para o crescimento econômico.

\subsection{Fatores Relacionados ao Surgimento das Fintechs}

Na revisão bibliográfica sobre FinTechs, é possível verificar a existência de pesquisas analisando os motivos e os fatores para o surgimento dessas instituições inovadoras. Por exemplo, Haddad e Hornuf (2018) verificaram maior formação de FinTechs quando os países são bem desenvolvidos e quando há 
pronta disponibilidade de capital de risco. Além disso, o número de servidores de Internet seguros e de assinaturas de telefonia móvel e a disponibilidade de mão-de-obra têm um impacto positivo no desenvolvimento desse novo segmento de mercado. Não menos importante, quanto maior é a dificuldade para as empresas acessarem empréstimos, maior será o número de FinTechs no país.

Haddad e Hornuf (2018) ainda concluem que há evidências de que a formação de FinTechs não ocorre necessariamente ao acaso, de modo que políticas pró-ativas podem influenciar o desenvolvimento deste novo setor, portanto com implicações para políticas públicas e reguladores do sistema financeiro e de pagamentos.

Outro exemplo de pesquisa que avalia os fatores para o surgimento de FinTechs é o trabalho de Zavolokina, Dolata e Schwabe (2016). Um dos fatores propostos envolve as mudanças na regulação das FinTechs. Segundo os autores, o interesse crescente no assunto demonstra a importância de um claro arcabouço legal para as atividades financeiras em que operam as FinTechs. Outros fatores considerados significativos incluem: conflito de agência, assimetria de informação e condições macroeconômicas instáveis. Os autores concluem que esses fatores, aliados aos avanços tecnológicos, não agem de modo exclusivo, mas são catalisadores para as atividades das FinTechs.

No entanto, ainda há uma carência de estudos que visem analisar a contribuição do surgimento das FinTechs para o crescimento econômico do país em que elas se instalam.

Nesse sentido, o objetivo deste trabalho é analisar como os fatores relacionados à formação das FinTechs em um determinado país podem contribuir para o seu o crescimento econômico, em especial observando o período pós-crise de 2008.

\section{RESULTADOS E DISCUSSÃO}

$\mathrm{Na}$ análise econométrica foi utilizado o nível de confiança de $95 \%(\alpha=0,05)$. Em todas as análises de variância, pode-se rejeitar a hipótese nula de que $\beta_{0}=\beta_{1}=\beta_{2}=\beta_{3}=\beta_{4}=0$ segundo o Teste-F, pois o valor P é muito baixo (próximo de zero) e F é maior do que F crítico uni-caudal.

Analisando-se as regressões lineares múltiplas realizadas, foram obtidos, por país, os resultados expressos na Tabela 5.

Em termos gerais, os resultados apontam para a utilidade do modelo econométrico proposto, baseado no modelo de crescimento endógeno schumpeteriano de um setor, pois o modelo foi considerado apropriado, pelo teste de significação, para os seis países da América Latina observados.

De modo mais específico, em nenhum dos países verificou-se uma relação significativa e positiva da "quantidade de FinTechs voltadas para a tecnologia Blockchain" com o crescimento econômico do país. Entretanto, cabe argumentar que essa tecnologia possivelmente ainda não proporcionou efetivamente uma evolução tecnológica e, portanto, de produtividade, nos países da América Latina que foram objeto do experimento.

Logo, esta variável não deveria ser descartada do modelo proposto, haja vista o potencial disruptivo da tecnologia Blockchain, que pode mudar radicalmente as relações de troca de informações entre os agentes do mercado financeiro e de pagamentos, em especial no contexto do Open Banking, em que os usuários assumem um papel central e as instituições, antes em posição de poder e detentoras dos dados dos clientes, passam a interagir, de forma segura e padronizada, para realizar suas operações.

Tabela 5 - Resultados das regressões lineares múltiplas, por país.

\begin{tabular}{|l|l|l|l|}
\hline País & Utilidade do modelo & $\begin{array}{l}\text { Fatores que contribuem para } \\
\text { o crescimento econômico }\end{array}$ & $\begin{array}{l}\text { Capacidade de explicação da } \\
\text { variabilidade do PIB per capita, } \\
\text { PPC (dólar), considerando somente } \\
\text { os fatores que contribuem para o } \\
\text { crescimento econômico }\end{array}$ \\
\hline Argentina & $\begin{array}{l}\text { Útil }(\mathrm{F} \text { de significação }= \\
0,004<0,05)\end{array}$ & $\begin{array}{l}\text { Quantidade de FinTechs } \\
\text { sediadas no país }\end{array}$ & $\begin{array}{l}53 \% \text { da variabilidade do PIB per } \\
\text { capita, } \text { PPC (dólar). }\end{array}$ \\
\hline
\end{tabular}




\begin{tabular}{|l|l|l|l|}
\hline Brasil & $\begin{array}{l}\text { Útil }(\mathrm{F} \text { de significação }= \\
0,0001<0,05)\end{array}$ & $\begin{array}{l}\text { Inverso do tempo médio de } \\
\text { existência das FinTechs } \\
\text { sediadas no país }\end{array}$ & $\begin{array}{l}\text { 62\% da variabilidade do PIB per } \\
\text { capita, } \text { PPC (dólar). }\end{array}$ \\
\hline Chile & $\begin{array}{l}\text { Útil }(\mathrm{F} \text { de significação }= \\
0,0007<0,05)\end{array}$ & $\begin{array}{l}\text { Quantidade de FinTechs } \\
\text { sediadas no país voltadas para } \\
\text { a tecnologia inteligência } \\
\text { artificial }\end{array}$ & $\begin{array}{l}\text { 70\% da variabilidade do PIB per } \\
\text { capita, } \text { PPC (dólar). }\end{array}$ \\
\hline Colômbia & $\begin{array}{l}\text { Útil }(\mathrm{F} \text { de significação }= \\
0,0003<0,05)\end{array}$ & $\begin{array}{l}\text { Inverso do tempo médio de } \\
\text { existência das FinTechs } \\
\text { sediadas no país }\end{array}$ & $\begin{array}{l}\text { 90\% da variabilidade do PIB per } \\
\text { capita, } \text { PPC (dólar). }\end{array}$ \\
\hline México & $\begin{array}{l}\text { Útil }(\mathrm{F} \text { de significação }= \\
0,0005<0,05)\end{array}$ & $\begin{array}{l}\text { Quantidade de FinTechs } \\
\text { sediadas no país }\end{array}$ & $\begin{array}{l}\text { 83\% da variabilidade do PIB } \text { per } \\
\text { capita, } \text { PPC (dólar). }\end{array}$ \\
\hline Peru & $\begin{array}{l}\text { Útil }(\mathrm{F} \text { de significação }= \\
0,002<0,05)\end{array}$ & $\begin{array}{l}\text { Inverso do tempo médio de } \\
\text { existência das FinTechs } \\
\text { sediadas no país }\end{array}$ & $\begin{array}{l}40 \% \text { da variabilidade do PIB } \text { per } \\
\text { capita } \text { PPC (dólar). }\end{array}$ \\
\hline
\end{tabular}

Apenas no caso do Chile houve relação significativa e positiva da "quantidade de FinTechs voltadas para a tecnologia inteligência artificial" com o crescimento econômico, o que pode denotar ainda um estágio inicial da inteligência artificial no que se refere aos seus efeitos sobre a produtividade e, por conseguinte, sobre o crescimento econômico. Por outro lado, tendo em vista a perspectiva de que a inteligência artificial pode provocar mudanças significativas nos postos de trabalhos existentes e na eficiência dos processos produtivos atuais, esta variável deveria permanecer no modelo para aumentar sua perenidade ou o seu poder futuro de predição, pois estaria mais sensível à evolução do mercado de trabalho e do processo produtivo que se avizinham.

Uma possível justificativa para a baixa capacidade de explicação do modelo para o caso do Peru é a reduzida quantidade de FinTechs naquele país, tanto de forma absoluta (17 FinTechs) como também em comparação com os demais países (apenas 4\% da quantidade de FinTechs do Brasil, por exemplo).

A "quantidade de FinTechs sediadas no país" foi variável significativa e de relação positiva com o crescimento econômico nos casos de Argentina e México, o que reforça a hipótese de que a existência de FinTechs instaladas no país contribui para o seu crescimento econômico.

A variável que em mais casos teve relação significativa e positiva com o crescimento econômico foi o "inverso do tempo médio de existência das FinTechs sediadas no país". Isso indica que quanto menor a média de tempo de vida das FinTechs instaladas no país, maior é o seu crescimento econômico, pois o fluxo significativo e crescente de novas FinTechs seria elemento que demonstra o caráter inovador do sistema financeiro e que proporciona maior desenvolvimento financeiro e que, por sua vez, favorece o crescimento econômico, desde que esse efeito não seja anulado por eventual taxa elevada de mortalidade dessas empresas. A variável "inverso do tempo médio de existência das FinTechs sediadas no país" foi a mais relevante para os casos do Brasil, Colômbia e Peru.

\section{CONSIDERAÇÕES FINAIS}

Neste trabalho analisou-se como os fatores relacionados ao surgimento de FinTechs nos países selecionados da América Latina influenciaram o crescimento econômico desses países após a crise financeira global de 2008. Foram selecionados os países da América Latina com maior relevância no uso de serviços oferecidos por FinTechs segundo o Índice Global de Adoção de FinTechs de 2019, elaborado pela Ernst \& Young (2019): Colômbia, Peru, México, Argentina, Chile e Brasil. Esses países representavam cerca de 91\% do mercado de FinTechs da América Latina em 2018 (BID, BID INVEST e FINNOVISTA, 2019, p. 28).

Um aspecto importante é o caráter teórico-empírico deste trabalho, pois visou reconhecer o advento das FinTechs e seu potencial disruptivo no âmbito do modelo de crescimento econômico schumpeteriano e segundo o conceito de "destruição criativa", bem como analisou possíveis efeitos do surgimento das FinTechs nesses países para o seu crescimento econômico.

Nesse sentido, o modelo de crescimento econômico endógeno schumpeteriano de um setor descrito por Aghion e Howitt (2009, p. 95) foi adaptado para considerar o setor de FinTechs como o elemento de produtividade relevante para o crescimento econômico. 

experimento.

Esse modelo matemático foi a base para a especificação do modelo econométrico e o desenho do

Na estimação dos parâmetros do modelo econométrico, a partir da observação do período de 2008 a 2018 - pós-crise de 2008 e após o advento das FinTechs - foram realizadas análises de como o PIB per capita, PPC (dólar), dos países selecionados da América Latina foi afetado por fatores relacionados ao surgimento de FinTechs nesses países.

Em termos gerais, os resultados apontam para a utilidade do modelo econométrico proposto, pois o modelo foi considerado apropriado, pelo teste de significação, para os seis países da América Latina observados.

Dentre os fatores relacionados ao surgimento de FinTechs, os mais relevantes para o crescimento econômico dos países foram o inverso do tempo médio de existência das FinTechs sediadas no país (3 de 6 países), a quantidade de FinTechs sediadas no país (2 de 6 países) e a quantidade de FinTechs voltadas para a tecnologia inteligência artificial (1 de 6 países).

Diante disso, no contexto estrito deste trabalho, pode-se considerar que o modelo de crescimento econômico de um setor baseado na teoria de Joseph Schumpeter e o conceito de "destruição criativa" são adequados para explicar o fenômeno das FinTechs.

Além disso, o modelo econométrico formulado foi significativo para toda a amostra de países da América Latina, de modo que é possível relacionar o surgimento de FinTechs nesses países com o crescimento econômico.

A partir dessas constatações, reforça-se a importância de se incentivar o surgimento de FinTechs nos países da América Latina como forma de impulsionar o seu crescimento econômico, portanto neste contexto as políticas públicas podem assumir um papel fundamental.

Países que possuem regulação de mercado mais flexível e que estabelecem melhor infraestrutura de suporte para transações seguras experimentam uma maior formação de FinTechs, pois, apesar do progresso de digitalização e globalização dos serviços financeiros, a decisão de onde instalar uma FinTech ainda é crucial (HADDAD e HORNUF, 2018, p. 95).

Portanto, há que se considerar a adoção de políticas públicas que incentivem a formação e a consolidação de FinTechs nos países da América Latina, pois este setor, que cresce de forma acelerada, pode contribuir para o crescimento econômico da região e, por conseguinte, melhorar o bem-estar da população e reduzir a pobreza.

\section{REFERÊNCIAS}

AGHION, P. Growth and Development: A Schumpeterian Approach. Annals of Economics and Finance, 5, n. 1, 2004. 1-25.

AGHION, P.; DURLAUF, S. N. Handbook of economic growth. Amsterdam: Elsevier, v. 1A, 2005.

AGHION, P.; HOWITT, P. Endogenous growth theory. Cambridge: MIT Press, 1998.

AGHION, P.; HOWITT, P. The Economics of Growth. Londres: The MIT Press, 2009.

AGHION, P.; JONES, B. F.; JONES, C. I. Artificial intelligence and economic growth. NBER Working Paper Series, Cambridge, MA, Outubro 2017. Disponivel em: <http://www.nber.org/papers/w23928>. Acesso em: 18 Março 2019.

AKDERE, Ç.; BENLI, P. The Nature of Financial Innovation: A Post-Schumpeterian Analysis. Journal of Economic Issues, v. 52, n. 3, p. 717-748, Setembro 2018.

ALT, R.; BECK, R.; SMITS, M. T. FinTech and the transformation of the financial industry. Electronic Markets, v. 28, p. 235-243, Agosto 2018. 
ASGARD. The Global Artificial Intelligence Landscape Database. Asgard, 2018. Disponivel em: <https://asgard.vc/global-ai/>. Acesso em: 16 Março 2019.

BANCO MUNDIAL. GDP per capita growth (annual \%). World Development Indicators Database, 2019. Disponivel em:

<https://data.worldbank.org/indicator/NY.GDP.PCAP.KD.ZG〉. Acesso em: 27 Maio 2019.

Licença: Creative Commons Attribution CC-BY 4.0.

BERKMEN, P. et al. Fintech in Latin America and the Caribbean: Stocktaking. IMF Working Paper, n. WP/19/71, Março 2019.

BID; BID INVEST; FINNOVISTA. Fintech na América Latina 2018: crescimento e consolidação. Banco Interamericano de Desenvolvimento. [S.1.], p. 140. 2019.

BRUE, S. L.; GRANT, R. R. História do pensamento econômico. São Paulo, SP: Cengage Learning, 2016. $608 \mathrm{p}$.

CRUNCHBASE INC. Companies. Crunchbase, 2019. Disponivel em:

<https://www.crunchbase.com/search/organization.companies/>. Acesso em: 17 Março 2019.

EATON, J.; KORTUM, S. Tecnology, Trade, and Growth: A Unifed Framework. European Economic Review, v. 45, n. 4-6, p. 742-755, Maio 2001.

ERNST \& YOUNG. Global FinTech Adoption Index. EYGM Limited. [S.1.], p. 44. 2019.

FERREIRA, P. C. et al. Desenvolvimento econômico: uma perspectiva brasileira. Rio de Janeiro: Elsevier, 2013.

FOSTER, A. D.; ROSENZWEIG, M. R. Microeconomics of Technology Adoption. Annual Review of Economics, v. 2, n. 1, p. 395-424, Março 2010.

GOMBER, P.; KOCH, J.-A. Digital Finance and FinTech: current research and future research directions. Journal of Business Economics, v. 87, n. 5, p. 537-580, Julho 2017.

HADDAD, C.; HORNUF, L. The emergence of the global fintech market: economic and technological determinants. Small Business Economics, v. 53, n. 1, p. 81-105, Junho 2018.

HAMDI, H.; HAKIMI, A.; SBIA, R. Finance and growth nexus: what role for institutions in developed and developing countries. Journal of Economic Development, v. 42, n. 4, p. 1-22, Dezembro 2017.

IOSCO Research Report on Financial Technologies (Fintech). International Organization of Securities Comissions. [Online]. 2017.

JONES, C. I.; VOLLRATH, D. Introdução à teoria do crescimento econômico. Tradução de Cristiana de Assis Serra. 3. ed. Rio de Janeiro: Elsevier, 2015.

KING, R. G.; LEVINE, R. Finance and Growth: Schumpeter Might be Right. The Quarterly Journal of Economics, v. 108, n. 3, p. 717-737, Agosto 1993. 
KRUGMAN, P. R.; OBSTFELD, M.; MELITZ, M. J. Economia internacional. Tradução de Ana Julia Perrotti-Garcia. São Paulo: Pearson Education do Brasil, 2015.

NAKAMOTO, S. Bitcoin: A Peer-to-Peer Electronic Cash System, 2008. Disponivel em: <https://bitcoin.org/bitcoin.pdf>. Acesso em: 18 Março 2019.

NICOLETTI, B. The Future of FinTech: Integrating Finance and Technology in Financial Services. Cham: Springer Nature, 2017.

OCAMPO, J.; FLORES, L. E. B. O desenvolvimento econômico da América Latina desde a independência. 1. ed. Rio de Janeiro: Elsevier, 2015.

OECD; ECLAC; CAF; EU. Latin American Economic Outlook 2019: Development in Transition. OECD Publishing. Paris. 2019.

PHILIPPON, T. The FinTech Opportunity. NBER Working Paper Series, Cambridge, MA, Agosto 2016. Disponivel em: <http://www.nber.org/papers/w22476>. Acesso em: 17 Março 2019.

RESENDE, M. F. D. C.; GONÇALVES, F. Uma extensão ao modelo Schumpeteriano de Crescimento Endógeno. Estud. Econ., São Paulo, v. 36, n. 1, p. 67-86, Mar 2006.

SARKINTUDU, S. M.; IBRAHIM, H. H.; ABDWAHAB, A. B. Taxonomy development of Blockchain platforms: Information systems perspectives. AIP Conference Proceedings. [Online]: AIP Publishing. 2018.

SCHUMPETER, J. A. Capitalism, Socialism and Democracy. 1 ${ }^{\mathrm{a}}$. ed. Londres: Routledge, 2013. $460 \mathrm{p}$.

SHIKIDA, P. F. A.; BACHA, C. J. C. Notas sobre o Modelo Schumpeteriano e suas principais correntes de pensamento. Teor. Evid. Econ., Passo Fundo, v. 5, n. 10, p. 107-126, maio 1998.

ZAVOLOKINA, L.; DOLATA, M.; SCHWABE, G. The FinTech phenomenon: antecedents of financial innovation perceived by the popular press. Financial Innovation, v. 2, n. 16, Dezembro 2016. 